\title{
Prevalencia de cardiopatías congénitas en una cohorte de 54.193 nacimientos entre 2011-2017
}

\author{
Prevalence of congenital heart disease in a cohort of 54,193 births between 2011-2017 \\ Lina M. Ibáñez-Correa ${ }^{1 *}$, Salomé Victoria ${ }^{1}$ y Paula Hurtado-Villa ${ }^{1,2}$ \\ ${ }^{1}$ Departamento de Ciencias Básicas de la Salud, Pontificia Universidad Javeriana Cali; ${ }^{2}$ Centro Médico Imbanaco. Cali, Colombia
}

\section{Resumen}

Objetivo: Describir la prevalencia de las cardiopatías congénitas en dos hospitales de Cali entre 2011-2017. Método: Se realizó un estudio retrospectivo de una cohorte de 54.193 nacimientos en dos hospitales de Cali, que incluyó recién nacidos desde el 1.. de enero 2011 hasta el 31 de diciembre de 2017 captados en el programa de vigilancia y seguimiento de defectos congénitos. Inicialmente se hizo un análisis descriptivo de los pacientes con cardiopatías y luego se analizó la relación de algunas variables con un chi-cuadrado $\left(\mathrm{X}^{2}\right)$ con una significancia de $p$-valor $<0,05$. Resultados: La prevalencia en esta cohorte fue de 2,42 por 1.000 nacimientos. De los 131 pacientes con cardiopatías congénitas, 73 (55,73\%) eran de sexo masculino; 91 (69,47\%) nacieron con peso adecuado para la edad gestacional y 31 (23,66\%) fueron pretérmino. De las madres, 30,53\% se encontraban entre 25 y 29 años y $42 \%$ eran primigrávidas. Respecto a las cardiopatías congénitas, la más frecuente fue la comunicación interventricular con 52 (32,30\%) casos; 105 (80,15\%) tenían una sola cardiopatía congénita y $62(47,33 \%)$ tenían cardiopatías aisladas. Las variables de peso para edad gestacional, edad materna y edad gestacional mostraron una relación estadísticamente significativa. Conclusiones: Las cardiopatías congénitas son de gran interés en salud pública dada su morbi-mortalidad y por ser causa de muerte en menores de un año en Colombia. Por lo tanto, se debe continuar trabajando en estrategias que mejoren su vigilancia, así como el diagnóstico prenatal, el tratamiento y el nivel de complejidad adecuado para cada paciente.

Palabras clave: Defectos congénitos. Cardiopatías congénitas. Prematuro. Bajo peso al nacer.

\section{Abstract}

Objective: To describe the prevalence of congenital heart disease in two hospitals of Cali in between 2011 and 2017. Method: A retrospective study of a cohort of 54,193 births was carried out in two hospitals of Cali, which included newborns from January 1, 2011 to December 31, 2017, captured through the surveillance program and monitoring of birth defects. Initially, a descriptive analysis of patients with congenital heart disease was performed, and the association of some variables with a chi-square $\left(\mathrm{X}^{2}\right)$ with a $p$-value significance $<0.05$. Results: The prevalence in this cohort was $2.42 \times 1,000$ births. Of the 131 patients with congenital heart disease, 73 (55.73\%) were male; 91 (69.47\%) were born with adequate weight for gestational age and 31 (23.66\%) were preterm. Of the mothers, 30.53\% were between 25 and 29 years old and $42 \%$ were primigravid. Regarding CC, the most frequent was interventricular communication with 52 (32.30\%) cases; 105 (80.15\%) had

\section{Correspondencia:}

*Paula Hurtado-Villa

E-mail: pmhurtadov@javerianacali.edu.co
Disponible en internet: 19-03-2021 Rev Colomb Cardiol. 2021;28(1):53-59 www.rccardiologia.com 0120-5633 / C 2020 Sociedad Colombiana de Cardiología y Cirugía Cardiovascular. Publicado por Permanyer. Este es un artículo open access bajo la licencia CC BY-NC-ND (http://creativecommons.org/licenses/by-nc-nd/4.0/). 
only one congenital heart disease and $62(47.33 \%)$ had isolated heart disease. The variables of weight for gestational age, maternal age and gestational age, showed a statistically significant association. Conclusions: Congenital cardiopathy is of great interest in public health, given their morbi-mortality and as a cause of death in children under 1 year old in Colombia. Therefore, we must continue to work on strategies that improve surveillance, as well as prenatal diagnosis, treatment and the level of complexity appropriate to each patient.

Key words: Birth defects. Congenital heart defect. Premature birth. Low birth weight.

\section{Introducción}

Las cardiopatías congénitas son alteraciones estructurales del corazón o de los grandes vasos que presentan -o potencialmente tienen el riesgo de presentar- un compromiso funcional ${ }^{1}$. Generalmente, se manifiestan entre la tercera y décima semanas de gestación, como consecuencia de las alteraciones del desarrollo embrionario del corazón².

Aunque la etiología de estas malformaciones aún no es clara, se considera que pueden tener una causa genética, ambiental o multifactorial, resultado de interacciones entre múltiples genes y factores ambientales $^{2,3}$. Sin embargo, prima la etiología multifactorial, lo que supone un aumento en el valor potencial de la investigación genética ${ }^{4}$. Adicionalmente, la mayoría de estas anomalías se produce de forma aislada, pero alrededor del $33 \%$ se encuentra asociada con otras malformaciones ${ }^{5}$.

Se estima una prevalencia mundial de cardiopatías congénitas de 0,5 a 9 por 1.000 nacidos vivos ${ }^{6}$, pero esta estimación varía de acuerdo con la edad de la población estudiada. Por ejemplo, si es antes del primer año de vida la prevalencia es de 8 por 1.000 niños y si es antes de los 16 años es de 12 por $1.000^{7}$. En Latinoamérica nacen cada año 54.000 niños con cardiopatías congénitas y de estos, 41.000 requieren algún tipo de tratamiento, pero infortunadamente solo son intervenidos $17.000^{8}$. En Colombia, el Ministerio de Salud describe una prevalencia de cardiopatías congénitas entre 7,5-9,5 por 1.000 nacimientos $^{9}$. Estos datos revelan un impacto significativo sobre la morbilidad, la mortalidad y el costo del sistema de salud en niños y adultos ${ }^{10}$, convirtiéndose en un evento de interés en salud pública. Por esta razón, con este estudio se busca describir la prevalencia de las cardiopatías congénitas en dos hospitales de la ciudad de Cali entre 2011 y 2017.

\section{Método}

Se realizó un estudio retrospectivo de una cohorte, en el que se incluyeron recién nacidos entre el $1 .^{\circ}$ de enero de 2011, hasta el 31 de diciembre de 2017, captados por el programa de vigilancia y seguimiento de defectos congénitos de la ciudad de Cali (se usó la metodología del Estudio Colaborativo Latinoamericano de Malformaciones Congénitas - ECLAMC) ${ }^{11}$, en el que se vigilaron 54.193 nacimientos en dos hospitales de la ciudad de Cali y se detectaron 131 recién nacidos con algunas cardiopatías congénitas.

El criterio de inclusión fue recién nacido con peso mayor a 500 gramos, nacidos vivos o muertos, con diagnóstico de cardiopatías congénitas al momento del nacimiento. El criterio de exclusión fue aquellas madres que no firmaron el consentimiento informado del programa. El diagnóstico de cardiopatías congénitas se realizó a través de los reportes de ecocardiograma del paciente que la madre tenía disponibles al momento de la valoración del paciente.

La información fue recolectada de la base de datos del programa de vigilancia y seguimiento de defectos congénitos de la ciudad de Cali, coordinado por la Pontificia Universidad Javeriana, con sede en esta misma ciudad. A las maternas que autorizaron el contacto telefónico para seguimiento en el consentimiento informado, se les llamó para conocer el estado de salud de los recién nacidos. El tiempo y la frecuencia con los que se hizo este seguimiento variaba de acuerdo con cada caso y con los planes de seguimiento de los pacientes propuestos en el programa de seguimiento de niños con anomalías congénitas en la ciudad de Bogotá12.

Inicialmente se hizo un análisis descriptivo de las variables de los pacientes con cardiopatías congénitas. La variable peso se categorizó como pequeño, adecuado y grande para la edad gestacional teniendo en cuenta la tabla de peso en gramos al nacer para ambos géneros propuesta por Montoya y Morales en el $2007^{13}$ : pequeño cuando este es inferior al percentil 10 de la distribución de los pesos correspondientes para la edad gestacional, adecuado cuando este se sitúa entre el percentil 10 y 90 de la distribución de los pesos correspondientes para la edad gestacional, y grande cuando es mayor al percentil 90 de la distribución de los pesos correspondientes para la edad gestacional ${ }^{14}$. 
La edad gestacional se clasificó como pretérmino $(<37$ semanas), a término (entre 37 y 41 semanas) y postérmino (42 o más semanas) $)^{14}$; gravidez como primigrávida (G1) y multigrávida (G2, G3, etc.); la edad materna se distribuyó en quinquenios de < 15 años, 15-19 años, 20-24 años. 25-29 años, 30-34 años, 35-39 años, 4045 años $y \geq 45$ años $y$, las cardiopatías congénitas fueron clasificadas en aisladas cuando no presentaban otras alteraciones cardíacas o extracardíacas, complejas cuando dos o más defectos cardíacos fueron descritos, asociadas cuando al menos una alteración menor o mayor extracardíaca fue reportada ${ }^{9}$ y sindrómicas cuando estaban relacionadas con un síndrome. Luego se analizó la asociación de algunas variables con un chi-cuadrado $\left(\mathrm{X}^{2}\right)$ con una significancia de $\mathrm{p}$-valor $<0,05$. El paquete estadístico utilizado fue EPI-INFO 7.0.

Por otro lado, de acuerdo con la resolución 008430 del 4 de octubre de 1993 del Ministerio de Salud de la República de Colombia, por la cual se establecen las normas científicas, técnicas y administrativas para la investigación en salud, este estudio se clasifica como investigación sin riesgo. La información de la cohorte se obtuvo previa firma de consentimiento informado por parte de la madre 0 del padre de cada paciente y posteriormente se hizo su inclusión en la base de datos. Los Comités de Ética e Investigación, de ambos hospitales aprobaron el estudio, el cual se encuentra bajo los lineamientos de la Declaración de Helsinki.

\section{Resultados}

Entre 2011 y 2017 se vigilaron 54.193 nacimientos en dos instituciones de la ciudad de Cali, de los cuales $1.389(2,56 \%)$ presentaron alguna malformación congénita (Tabla 1). Del total de pacientes con malformaciones congénitas, 131 casos tenían una o varias cardiopatías congénitas, por lo cual la prevalencia en esta cohorte fue de 2,42 x 1.000 nacimientos.

Al analizar las características de los 131 recién nacidos con cardiopatías congénitas durante el periodo de estudio, se encontró mayor cantidad de casos en el año 2014 y 2016, con 27 reportes (20,61\%) cada uno. Del total de los pacientes con cardiopatías congénitas $73(55,73 \%)$ eran de sexo masculino, $57(43,51 \%)$ de sexo femenino y $1(0,76 \%)$ indeterminado (Tabla 2$)$. El promedio del peso al nacer fue de 2803,37 gramos (desviación estándar $-\mathrm{DE}=875,89$, mínimo $=560$ y máximo $=4.352$ gramos) y al clasificar esta variable, los más frecuentes fueron los recién nacidos con peso
Tabla 1. Nacimientos vigilados con y sin malformaciones en dos instituciones de la ciudad de Cali durante 2011 a 2017

\begin{tabular}{|l|c|c|}
\hline Malformados & $\mathbf{n}$ & $\%$ \\
\hline No & 52.804 & 97,44 \\
\hline Sí & 1.389 & 2,56 \\
\hline Total & 54.193 & 100,00 \\
\hline
\end{tabular}

Tabla 2. Características de los recién nacidos con cardiopatías congénitas nacidos en dos instituciones de la ciudad de Cali durante 2011 a 2017

\begin{tabular}{|c|c|c|}
\hline Características de los recién nacidos & n (131) & $\%$ \\
\hline $\begin{array}{l}\text { Año de nacimiento } \\
2011 \\
2012 \\
2013 \\
2014 \\
2015 \\
2016 \\
2017\end{array}$ & $\begin{array}{c}15 \\
12 \\
9 \\
27 \\
16 \\
27 \\
25\end{array}$ & $\begin{array}{c}11,45 \\
9,16 \\
6,87 \\
20,61 \\
12,21 \\
20,61 \\
19,08\end{array}$ \\
\hline $\begin{array}{l}\text { Sexo } \\
\text { Femenino } \\
\text { Masculino } \\
\text { Indeterminado }\end{array}$ & $\begin{array}{c}57 \\
73 \\
1\end{array}$ & $\begin{array}{c}43,51 \\
55,73 \\
0,76\end{array}$ \\
\hline $\begin{array}{l}\text { Peso para edad gestacional } \\
\text { Pequeño } \\
\text { Adecuado } \\
\text { Grande } \\
\text { No aplica } \\
\text { Sin información }\end{array}$ & $\begin{array}{l}21 \\
91 \\
17 \\
1 \\
1\end{array}$ & $\begin{array}{l}16,03 \\
69,47 \\
12,98 \\
0,76 \\
0,76\end{array}$ \\
\hline $\begin{array}{l}\text { Estado al nacer } \\
\text { Fallecido } \\
\text { Vivo }\end{array}$ & $\begin{array}{c}5 \\
126\end{array}$ & $\begin{array}{c}3,82 \\
96,18\end{array}$ \\
\hline $\begin{array}{l}\text { Estado a la salida del hospital } \\
\text { Fallecido } \\
\text { Sin salida } \\
\text { Vivo }\end{array}$ & $\begin{array}{c}8 \\
16 \\
107\end{array}$ & $\begin{array}{c}6,11 \\
12,21 \\
81,86\end{array}$ \\
\hline $\begin{array}{l}\text { Prematurez } \\
\text { Sin información } \\
\text { Término } \\
\text { Pretérmino }\end{array}$ & $\begin{array}{c}1 \\
99 \\
31\end{array}$ & $\begin{array}{c}0,76 \\
75,57 \\
23,66\end{array}$ \\
\hline
\end{tabular}

adecuado para la edad gestacional con 91 (69,47\%) casos (Tabla 2).

Durante el parto nacieron vivos $126(96,18 \%)$ y en el momento del alta egresaron vivos 107 (81,86\%), 8 fallecidos $(6,11 \%)$ y a $16(12,21 \%)$ aún no se les había dado alta (Tabla 1). Respecto a la edad gestacional, el número promedio de semanas fue de $37(\mathrm{DE}=4,05$; mínimo $=20$ y máximo $=41$ semanas). Al categorizarlos como pretérmino, a término y postérmino, se encontró 
Tabla 3. Características de las madres de los recién nacidos con cardiopatías congénitas de dos instituciones de la ciudad de Cali durante 2011 y 2017

\begin{tabular}{|l|c|c|}
\hline Características maternas & n (131) & $\%$ \\
\hline $\begin{array}{l}\text { Edad materna } \\
<15\end{array}$ & 1 & \\
$15-19$ & 17 & 0,76 \\
$20-24$ & 21 & 12,98 \\
$25-29$ & 40 & 16,03 \\
$30-34$ & 21 & 30,53 \\
$35-39$ & 17 & 16,03 \\
$40-44$ & 13 & 12,98 \\
$>=45$ & 1 & 0,92 \\
\hline Gravidez & & \\
\hline Primigrávida & & \\
Multigrávida & 55 & 41,98 \\
& 76 & 58,02 \\
\hline
\end{tabular}

que 99 pacientes $(75,57 \%)$ nacieron a término, 31 $(23,66 \%)$ fueron pretérmino y $1(0,76)$ estaba sin información (Tabla 2).

En cuanto a las madres de los recién nacidos con cardiopatías congénitas, la edad promedio fue de 28 años ( $D E=7,58$; mínimo $=14$ años y máximo= 49 años) y el $30,53 \%$ se encontraban en el grupo etario comprendido entre los 25 y 29 años de edad, siendo lo más frecuente (Tabla 3). El número de embarazos promedio fue de $2(\mathrm{DE}=1,17 ;$ mínimo= 1 y máximo= 6 embarazos); las mujeres multigrávidas fueron los casos más frecuentes, con $58,02 \%$ (tabla 3 ).

Al analizar los diagnósticos de cardiopatías congénitas que presentaban los 131 casos, se encontró que $105(80,15 \%)$ presentaban un solo diagnóstico y los demás, dos o más (Tabla 4). Por tanto, al consolidar todos estos diagnósticos, en total hay 161 cardiopatías congénitas, de entre las cuales las más frecuentes fueron la comunicación interventricular (CIV) y la comunicación interauricular (CIA), con $52(32,30 \%)$ y 41 $(25,47)$ casos, respectivamente (Tabla 5).

Adicionalmente, se analizó la clasificación de las cardiopatías congénitas y se encontró que 62 (47,33\%) eran aisladas, 24 (18,32\%) asociadas, 23 (17,56\%) complejas y $22(16,69 \%)$ sindrómicas (Tabla 6). Entre los síndromes que se hallaron están: Down con 16 (72,73\%), Edwards $1(4,55 \%)$ y Klinefelter 1 (4,55\%) (Tabla 7$)$.

De los 131 casos con cardiopatías congénitas, tan sólo $13(9,92 \%)$ tenían diagnóstico prenatal, mientras que 48 $(36,64 \%)$ no lo tenían, y, aún más preocupante, 70 $(53,44 \%)$ no tenía esta información (Tabla 8). Adicionalmente, de estos 131 se hizo seguimiento a 126 casos, logrando contacto con $68(53,97 \%)$ de las madres o familiares de los recién nacidos. De estos, 54 (79,41\%)
Tabla 4. Número de cardiopatías congénitas encontradas en los 131 casos identificados y nacidos en dos instituciones de la ciudad de Cali durante 2011 a 2017

\begin{tabular}{|c|c|c|}
\hline $\begin{array}{l}\text { Número de cardiopatías congénitas por caso y } \\
\text { descripción de la(s) malformación(es) }\end{array}$ & $\mathrm{n}$ & $\%$ \\
\hline $\begin{array}{l}\text { Una malformación } \\
\text { CIV }^{\text {a }} \\
\text { CIA }^{\text {b }} \\
\text { Cardiopatía no especificada } \\
\text { Otras cardiopatías } \\
\text { Tetralogía de Fallot } \\
\text { Hipoplasia corazón izquierdo } \\
\text { Anomalía de la arteria pulmonar } \\
\text { Transposición de grandes vasos } \\
\text { Coartación de aorta } \\
\text { Ductus arterioso persistente } \\
\text { Anomalía de la válvula tricúspide } \\
\text { Otras anomalías cono-troncales } \\
\text { Ventrículo único }\end{array}$ & $\begin{array}{l}105 \\
34 \\
23 \\
13 \\
8 \\
8 \\
5 \\
4 \\
3 \\
2 \\
2 \\
1 \\
1 \\
1\end{array}$ & $\begin{array}{r}80,15 \\
32,38 \\
21,90 \\
12,38 \\
7,62 \\
7,62 \\
4,76 \\
3,81 \\
2,86 \\
1,90 \\
1,90 \\
0,95 \\
0,95 \\
0,95\end{array}$ \\
\hline $\begin{array}{l}\text { Dos malformaciones } \\
\text { CIA + CIV } \\
\text { CIA + Ductus arterioso persistente } \\
\text { CIA + coartación de aorta } \\
\text { Hipoplasia corazón izquierdo + coartación de } \\
\text { aorta } \\
\text { CIV + ductus arterioso persistente } \\
\text { Anomalía de la pulmonar + CIV } \\
\text { Ductus arterioso persistente + Otras } \\
\text { cardiopatías } \\
\text { Anomalía de la tricúspide + Anomalía de la } \\
\text { pulmonar } \\
\text { Transposición de grandes vasos }\end{array}$ & $\begin{array}{c}22 \\
11 \\
2 \\
2\end{array}$ & $\begin{array}{c}16,79 \\
50,00 \\
9,10 \\
9,10\end{array}$ \\
\hline $\begin{array}{l}\text { Tres malformaciones } \\
\text { CIV + CIA + Otras cardiopatías } \\
\text { Hipoplasia corazón izquierdo + Anomalía de la } \\
\text { aorta + Anomalía de la mitral } \\
\text { Transposición de grandes vasos }\end{array}$ & $\begin{array}{l}4 \\
2 \\
1\end{array}$ & $\begin{array}{c}3,05 \\
50,00 \\
25,00\end{array}$ \\
\hline
\end{tabular}

a Comunicación interventricular; b Comunicación interauricular.

seguían vivos y 14 (20,59\%) habían fallecido y, de acuerdo con la información obtenida en el seguimiento, se considera que en $10(71,43 \%)$ de los 14 recién nacidos fallecidos, la muerte estuvo relacionada con las cardiopatías.

Finalmente, en la relación se encontró que el peso para la edad gestacional, la edad materna y la edad gestacional son estadísticamente significativas con un p-valor $<0,05$ (Tabla 9).

\section{Discusión}

La prevalencia de cardiopatías congénitas encontrada en este estudio $(2,42 \times 1.000)$ es mayor que la descrita en una cohorte previa que incluyó 44.985 nacimientos en Colombia (1,2 por 1.000 nacidos vivos) ${ }^{9}$, pero menor a la reportada en otros estudios, como el de Costa Rica que describe una prevalencia de 6,1 por 
Tabla 5. Cardiopatías congénitas consolidadas de los 131 casos identificados y nacidos en dos instituciones de la ciudad de Cali durante 2011 a 2017

\begin{tabular}{|l|c|c|}
\hline Malformación cardíaca & n & $\%$ \\
\hline CIV & 52 & 32,30 \\
\hline CIA $^{b}$ & 41 & 25,47 \\
\hline Cardiopatía no especificada & 13 & 8,07 \\
\hline Otras cardiopatías & 11 & 6,83 \\
\hline Tetralogía de Fallot & 8 & 4,97 \\
\hline Hipoplasia corazón izquierdo & 7 & 4,35 \\
\hline Anomalía de la pulmonar & 6 & 3,73 \\
\hline PDA ductus arterioso persistente & 6 & 3,73 \\
\hline Transposición grandes vasos & 6 & 3,73 \\
\hline Coarctación de la aorta & 5 & 3,11 \\
\hline Anomalía de la válvula tricúspide & 2 & 1,24 \\
\hline Anomalía de la aorta & 1 & 0,62 \\
\hline Anomalía de la válvula mitral & 1 & 0,62 \\
\hline Otras anomalías cono-troncales & 1 & 0,62 \\
\hline Ventrículo único & 1 & 0,62 \\
\hline Total & 161 & 100,00 \\
\hline a Comunicación interventricular; b Comunicación interauricular. & \\
\hline
\end{tabular}

Tabla 6. Cardiopatías congénitas aisladas y sindrómicas en la ciudad de Cali de 2011-2017

\begin{tabular}{|l|c|c|}
\hline Clasificación de cardiopatías congénitas & n & $\%$ \\
\hline Aislada & 62 & 47,33 \\
\hline Asociada & 24 & 18,32 \\
\hline Compleja & 23 & 17,56 \\
\hline Sindrómica & 22 & 16,79 \\
\hline Total & 131 & 100,00 \\
\hline
\end{tabular}

1.000 nacimientos (IC95\%: 5,6-6,7) ${ }^{15}$. En Quebec, la prevalencia de cardiopatías congénitas en el primer año de vida fue de 13,1 por cada 1.000 nacidos vivos ${ }^{16}$ y los registros europeos describen una prevalencia total de 8 por 1,000 nacidos vivos ${ }^{4}$. Es probable que la prevalencia encontrada en esta investigación esté subestimada, pues hay estudios que han demostrado que algunas cardiopatías pueden permanecer asintomáticas varios meses después del egreso hospitalario ${ }^{17}$.
Tabla 7. Síndromes relacionados con cardiopatías congénitas en la ciudad de Cali de 2011-2017

\begin{tabular}{|l|c|c|}
\hline \multicolumn{3}{|c|}{ Cardiopatías sindrómicas } \\
\hline Síndrome de Down & n & $\%$ \\
\hline Síndrome de Edwards & 16 & 72,73 \\
\hline Síndrome de Klinefelter & 1 & 4,55 \\
\hline Anomalía cromosómica & 1 & 4,55 \\
\hline Síndrome de Vacterl & 1 & 4,55 \\
\hline Síndrome de rubéola congénita & 1 & 4,55 \\
\hline Otros síndromes especificados & 1 & 4,55 \\
\hline Total & 1 & 4,55 \\
\hline
\end{tabular}

Tabla 8. Diagnóstico prenatal de las cardiopatías congénitas en Cali de 2011 a 2015

\begin{tabular}{|l|c|c|}
\hline Diagnóstico prenatal de la cardiopatías congénitas & $\mathbf{n}$ & $\%$ \\
\hline Si & 13 & 9,92 \\
\hline No & 48 & 36,64 \\
\hline Sin información & 70 & 53,44 \\
\hline Total & 131 & 100,00 \\
\hline
\end{tabular}

Las cardiopatías más comunes fueron los defectos de tabique interauricular e interventricular (CIA y CIV), lo cual concuerda con lo reportado en la literatura ${ }^{17}$, pero otros estudios muestran el conducto arterioso permeable como la más frecuente, seguida de la comunicación interauricular. En prematuros, el conducto arterioso permeable fue la afección que más se presentó, mientras que en los recién nacidos a término las que predominaron fueron la $\mathrm{CIA}$ y la $\mathrm{CIV}^{18}$.

En lo concerniente a la edad materna promedio, la cual es de 28 años, se encuentra dentro del rango reportado por otros estudios, con una relación estadísticamente significativa. Sin embargo, es importante anotar que hay un mayor riesgo de cardiopatías congénitas sindrómicas en madres mayores de 34 años y de cardiopatías congénitas aisladas o sindrómicas en madres menores de 15 años $^{15}$.

De acuerdo con los hallazgos de otro estudio ${ }^{18}$ la frecuencia de cardiopatías congénitas era mayor en los prematuros. En nuestro estudio, sin embargo, el $75,57 \%$ de los pacientes con cardiopatía nació a 
Tabla 9. Relación de las variables peso para edad gestacional, gravidez, edad materna y edad gestacional con cardiopatías congénitas

\begin{tabular}{|c|c|c|c|c|}
\hline \multirow[t]{2}{*}{ Variables } & \multirow[t]{2}{*}{ Categoría de las variables } & \multicolumn{2}{|c|}{ Cardiopatía congénita } & \multirow[t]{2}{*}{ P-valor } \\
\hline & & No (\%) & Si (\%) & \\
\hline \multirow[t]{5}{*}{ Peso } & Pequeño & $3.427(6,32)$ & $21(0,04)$ & \multirow[t]{5}{*}{$0,00^{*}$} \\
\hline & Adecuado & $42.241(77,95)$ & $91(0,17)$ & \\
\hline & Grande & $8.322(15,36)$ & $17(0,03)$ & \\
\hline & No aplica & $49(0,09)$ & $1(0,01)$ & \\
\hline & Sin información & $23(0,04)$ & $1(0,01)$ & \\
\hline \multirow[t]{3}{*}{ Gravidez } & Multigrávida & $27.427(50,61)$ & $76(0,14)$ & \multirow[t]{3}{*}{0,24} \\
\hline & Primigrávida & $26.599(49,08)$ & $55(0,10)$ & \\
\hline & Sin información & $36(0,07)$ & $0(0,00)$ & \\
\hline \multirow[t]{9}{*}{ Edad materna } & $<15$ & $218(0,40)$ & $1(0,00)$ & \multirow[t]{9}{*}{$0,00^{*}$} \\
\hline & $15-19$ & $6.863(12,66)$ & $17(0,03)$ & \\
\hline & $20-24$ & $15.094(27,85)$ & $21(0,04)$ & \\
\hline & $25-29$ & $15.238(28,12)$ & $40(0,07)$ & \\
\hline & $30-34$ & $10.481(19,34)$ & $21(0,04)$ & \\
\hline & $35-39$ & $4.895(9,03)$ & $17(0,03)$ & \\
\hline & $40-44$ & $1.144(2,11)$ & $13(0,02)$ & \\
\hline & $\geq 45$ & $118(0,22)$ & $1(0,00)$ & \\
\hline & Sin información & $11(0,02)$ & $0(0,00)$ & \\
\hline \multirow[t]{4}{*}{ Edad gestacional } & Pretérmino & $4.546(8,39)$ & $31(0,06)$ & \multirow[t]{4}{*}{$0,00^{*}$} \\
\hline & Término & $49.475(91,29)$ & $99(0,18)$ & \\
\hline & Postérmino & $28(0,05)$ & $0(0,00)$ & \\
\hline & Sin información & $13(0,02)$ & $1(0,00)$ & \\
\hline
\end{tabular}

* Relación estadísticamente significativa ( $p$-valor $<0,05$ ).

término y tan sólo el 23,66\% fueron pretérmino. Al hacer el análisis de asociación con edad gestacional, se mostró que esta era estadísticamente significativa, al igual que con el peso para la edad gestacional.

En esta cohorte, $26(19,84 \%)$ pacientes con cardiopatías congénitas presentaron dos o tres malformaciones cardiacas, por lo cual es fundamental tener un personal médico idóneo en la búsqueda de estas enfermedades, tanto en el período prenatal como en el postnatal. Sólo el 9,92\% de los pacientes tuvo diagnóstico prenatal, dato que se convierte en información valiosa para la toma de decisión por parte de los padres, acerca de la evolución de la gestación y la preparación psicológica de estos ${ }^{19}$. Varios estudios han demostrado que para mejorar la detección prenatal de cardiopatías congénitas se debe integrar la ecocardiografía fetal al examen obstétrico rutinario ${ }^{20}$.

\section{Conclusiones}

Las cardiopatías congénitas son defectos congénitos que impactan tanto la morbilidad como la mortalidad de los niños, y esto las hace de gran interés en salud pública. En este estudio, se encontró que los niños con cardiopatías congénitas eran producto de la primera gestación, de madres entre 25 y 29 años y en su mayoría presentaron cardiopatías congénitas aisladas. De los pacientes en quienes se logró un seguimiento, el $15 \%$ había fallecido a causa de la cardiopatía congénita. Por ello se debe continuar trabajando en 
estrategias que mejoren la vigilancia, el diagnóstico, el tratamiento y el seguimiento para cada paciente.

Adicionalmente, es importante mejorar el diagnóstico prenatal, ya que esto permitiría tomar decisiones apropiadas en el momento oportuno, lo cual tendría un gran impacto en la morbimortalidad infantil.

\section{Agradecimientos}

Los autores agradecen a las instituciones de la ciudad de Cali que hacen parte del programa de vigilancia y seguimiento de defectos congénitos, así como a los pacientes y sus familias.

\section{Financiamiento}

Pontificia Universidad Javeriana - Cali.

\section{Conflicto de intereses}

Los autores declaran no tener ningún conflicto de intereses, ya que son independientes respecto a las instituciones financiadoras y de apoyo.

\section{Responsabilidades éticas}

Protección de personas y animales. Los autores declaran que para esta investigación no se han realizado experimentos en seres humanos ni en animales.

Confidencialidad de los datos. Los autores declaran que han seguido los protocolos de su centro de trabajo sobre la publicación de datos de pacientes.

Derecho a la privacidad y consentimiento informado. Los autores declaran que en este artículo no aparecen datos de pacientes.

\section{Bibliografía}

1. Madrid AJ, Restrepo JP. Cardiopatías Congénitas. Revista Gastrohnup. 2013;15(1):S56-S72.

2. Perich-Durán RM. Cardiopatías congénitas más frecuentes y seguimiento en atención primaria. Pediatr Integral. 2012;XVI(8):622-35.

3. Nora JJ. Multifactorial inheritance hypothesis for the etiology of congenital heart diseases. The genetic-environmental interaction. Circulation. 1968;38:604-17 [PMID: 4876982 DOI: 10.1161/01. CIR.38.3.604].

4. Grosse SD, Khoury MJ. What is the clinical utility of genetic testing? Genet Med. 2006;8:448-450 [PMID: 16845278 DOI: 10.109701. gim.0000227935.26763.c6].

5. Noonan JA, Ehmke DA. Associated non cardiac malformations in children with congenital heart disease. J Pediatr. 1963;63:468-71.

6. Sandoval N. Cardiopatías congénitas en Colombia y en el mundo. Rev Colomb Cardiol. 2015;22(1):1-2.

7. Roy DL, McIntyre L, Human DG, Nanton MA, Sherman GJ, Allen LM, et al. Trends in the prevalence of congenital heart disease: comprehensive observations over a 24 -year period in a defined region of Canada. Can J Cardiol, 1994;10:821-6.

8. Groisman B, Bidondo M, Barbero P, Gil J, Liascovich R, Grupo Renac. RENAC: Registro Nacional de Anomalías Congénitas de Argentina. Arch Argent Pediatr. 2013;111(6):484-94

9. Batalxe E, Zarante I. Prevalencia de las malformaciones cardiacas congénitas en 44.985 nacimientos en Colombia. Arch Cardiol Mex. 2006;76(3):263-8.

10. American Heart Association. Statistical Fact Sheet 2015 Update. Congenital Heart Defects. [Acceso 14 Mar 2017]. Disponible en https://www. heart.org/idc/groups/heart-public/@wcm/@sop/@smd/documents/downloadable/ucm462018.pdf.

11. ECLAMC. Estudio Colaborativo Latinoamericano de Malformaciones Congénitas. Manual Operacional. Edición 2009.

12. Zarante I, Sarmiento K, Mallarino C, Gracia G. Description of Bogotá Birth Defects Surveillance and Follow-up Program. Journal of Registry Management. 2016;41(3):116-21.

13. Montoya-Restrepo NE, Correa-Morales JC. Curvas de peso al nacer. Rev. Salud Pública. 2007:9(1):1-10.

14. Gómez-Gómez M, Danglot-Banck C, Aceves-Gómez M. Clasificación de los recién nacidos. Revista Mexicana de Pediatría. 2012;79(1):32-9.

15. Benavides A, Faerron J, Umaña L, Romero J. Epidemiología y registro de las cardiopatías congénitas en Costa Rica. Rev Panam Salud Publica. $2011 ; 30(1): 31-8$

16. Marelli AJ, Ionescu-Ittu R, Mackie AS, Guo L, Dendukuri N, Kaouache M. Lifetime Prevalence of Congenital Heart Disease in the General Population From 2000 to 2010. Circulation. 2014;130:749-756. http://circ. ahajournals.org/content/130/9/749.full.

17. Viñals F, Giuliano A. Cardiopatías congénitas. Incidencia postnatal (II)*. Rev Chil Obstet Ginecol. 2002;67(3):207-10.

18. Mendieta G, Alcántara E, Mendieta H, Dorantes R, Ortiz G, Otero G. Incidencia de las cardiopatías congénitas y los factores asociados a la letalidad en niños nacidos en dos hospitales del Estado de México. Gaceta Médica de México. 2013;149:617-23.

19. Crawford DC, Chita SK, Allan LD. Prenatal detection of congenital heart disease: Factors affecting obstetric management and survival. Am J Obstet Gynecol. 1988;159:352-6.

20. Viñals $F$, Giuliano A. Cardiopatías congénitas. Incidencia antenatal. Rev Chil Obstet Ginecol. 2002;67(3):203-6. 